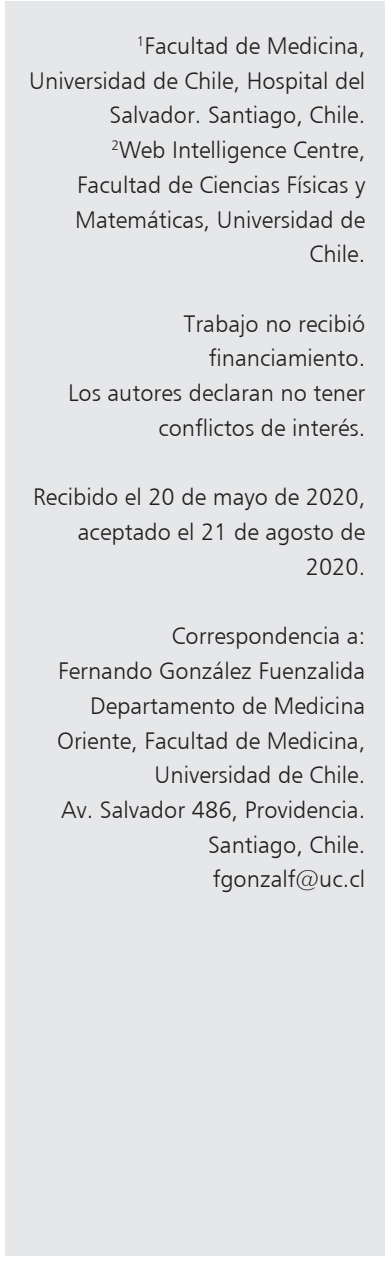

\section{Epidemias en la actualidad: de cómo los modelos matemáticos y estadísticos permiten entenderlas, aún a profesionales “adversos" a ellos}

\author{
FERNANDO GONZÁLEZ FUENZALIDA ${ }^{1}$, \\ FRANCISCA GONZÁLEZ COHENS ${ }^{2}$
}

\section{Mathematical models to understand pandemics}

Since the declaration of SARS CoV-2 pandemic, we have witnessed an accelerated increase in new cases, frequently associated with the need for intensive care and mechanical ventilation. In parallel, we have been invaded by many experts in the press and who expose their knowledge on the behavior of epidemics who use concepts that are not always well understood by the medical community. Some concepts should be knowledgeable to understand the epidemic spread. First, the epidemic spread description is not modeled with an exponential curve, but rather with a Gompertz curve. Second, a gamma curve describes the period of contagiousness. Third, the contagion magnitude or rate can be calculated and modeled. Explaining these mathematical concepts in a simple and graphic way will allow readers to understand better what is happening with the current pandemic.

(Rev Med Chile 2021; 149: 422-432)

Key words: Basic Reproduction Number; Models, Theoretical; Communicable Diseases; Pandemics.

\footnotetext{
E
} 131 de diciembre de 2019 la Organización Mundial de la Salud (OMS) reportó pacientes enfermos de una neumonía de etiología desconocida en Wuhan, China ${ }^{1}$. Más tarde se explicó era producida por un nuevo beta-coronavirus ${ }^{2}$. Semanas después, Tailandia comunicó de más pacientes infectados ${ }^{3} \mathrm{y}$ así sucesivamente otros países hasta que, el 30 de enero la OMS declara que la infección por este Coronavirus, llamado SARS-CoV-2 (Severe Acute Respiratory Syndrome Coronavirus-2) y la neumonía secundaria, COVID-19 (Coronavirus disease 2019) se había convertido en pandemia ${ }^{3}$.

El primer caso chileno apareció el 3 de marzo $y$, a contar de esa fecha, hemos sido testigos de un aumento progresivo de casos hasta 12.858 al 25 de abril $^{4}$. En paralelo a las múltiples iniciativas de salud pública emanadas del Ministerio de Salud, la prensa ha inundado la opinión pública con diversos expertos que utilizan términos técnicos para explicar el curso de las epidemias, establecer pronósticos de impacto sobre la sociedad y atisbar futuros más o menos sombríos sobre lo que podría esperarse de esta infección hasta que surjan vacunas o antivirales que permitan controlarla.

Nuestro objetivo es explicar los conceptos matemáticos descriptivos del curso de epidemias, de tal forma de aclarar conceptos básicos que no se enseñan habitualmente en las universidades y que en estos momentos resultan de muchísima utilidad para la comunicación y desarrollo de planes de acción. 


\section{El agente infeccioso y período de contagiosidad}

Cuando aparece un agente infeccioso transmisible ante el cual no existe inmunidad poblacional, el primer infectado contagiará a varios otros y éstos, a su vez, a otros cuantos más y así sucesivamente hasta contagiar a una fracción o a toda la población.

Tras el contacto con el agente, existe un período de incubación durante el cual éste se multiplica y un período donde aparece su capacidad de contagiar a otros, por ejemplo, por secreciones respiratorias o contacto sanguíneo. Ambos fenómenos pueden describirse matemáticamente usando una distribución de tipo gama (Figura 1), que ilustra el comportamiento de la multiplicación del virus y su contagiosidad en el tiempo, que es mayor al principio y disminuye en paralelo con la resolución de la infección y, muchas veces, también con los síntomas. De ello se desprende que las medidas mitigatorias de la propagación serán más eficaces al principio de la infección.

Esta distribución es descrita por dos parámetros: Alfa, que muestra la máxima intensidad de probabilidad y da la forma de la distribución y por Beta, que determina la forma o alcance de la asimetría positiva desplazando la densidad de probabilidad en la cola derecha ${ }^{5}$.

Las Figuras 2 y 3 ilustran esta función aplicada a enfermedades infecciosas. Los períodos de contagiosidad serían 8-9 días para influenza y 40 para ébola, aunque cabe la posibilidad de períodos más prolongados. Este mayor lapso puede ser difícil de determinar con exactitud; por ejemplo, si bien puede detectarse virus Zika en el semen hasta 120 días (100-139 días) después de la infección, no se han reportado casos de infección por esta vía más allá de 44 días $^{6}$.

\section{Comportamiento del Crecimiento de la Curva de Infectados}

El índice reproductivo básico de la enfermedad se denomina R0, definido como el número promedio de casos nuevos que genera un caso dado a lo largo de un período infeccioso ${ }^{9}$. Un valor $\mathrm{R} 0<1$ implica baja contagiosidad y R $0>1$ enfermedades que tenderán a propagarse, tales como sarampión (R0: 12-18), viruela (4-5), Zika (2,06), VIH (2-5), influenza "española" $(1,5-3,8)$, Ébola $(1,5)^{10}$.

El R0 del SARS-CoV-2 es $1,45-2,5^{11}$, pero, veremos más adelante que ese número puede ser bastante variable y depende de cuándo y cómo se lo calcule ${ }^{10}$.

Un R0 > 1, por ejemplo 2, en una población $100 \%$ susceptible significa que cada caso nuevo contagiará a otros dos y éstos a otros dos adicionales cada uno, lo que intuitivamente se asocia a una descripción matemática como curvas de crecimiento exponencial con una función $y=e^{x}$, donde " $y$ " es el número de casos acumulados, "e" el número de Euler $(2,71828)$ y " $x$ " el momento de medición.

Las proyecciones iniciales del número de contagios acumulados por SARS-CoV-2, y destacadas

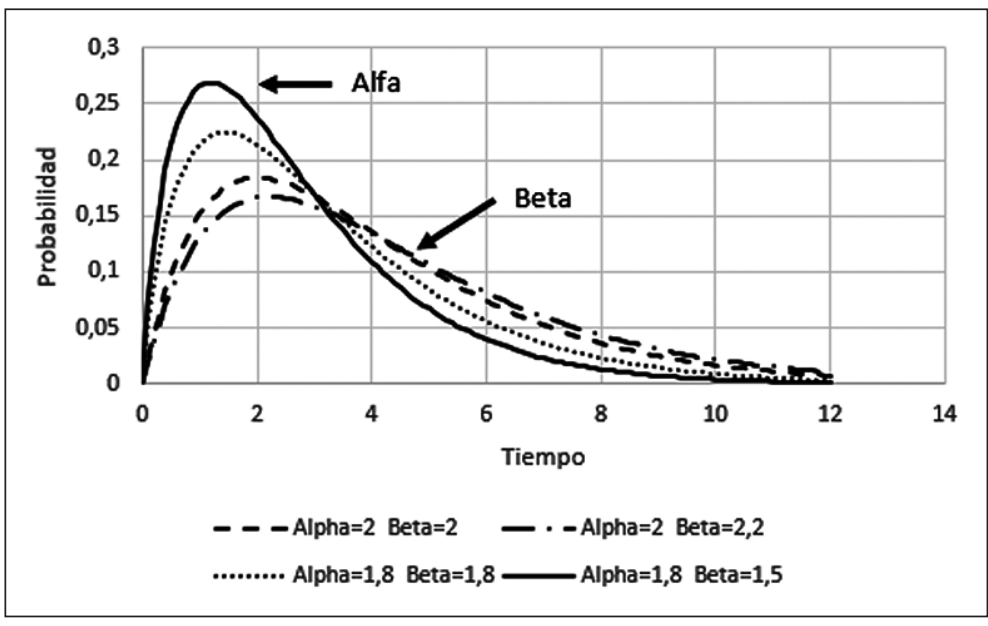

Figura 1. Función gama. 


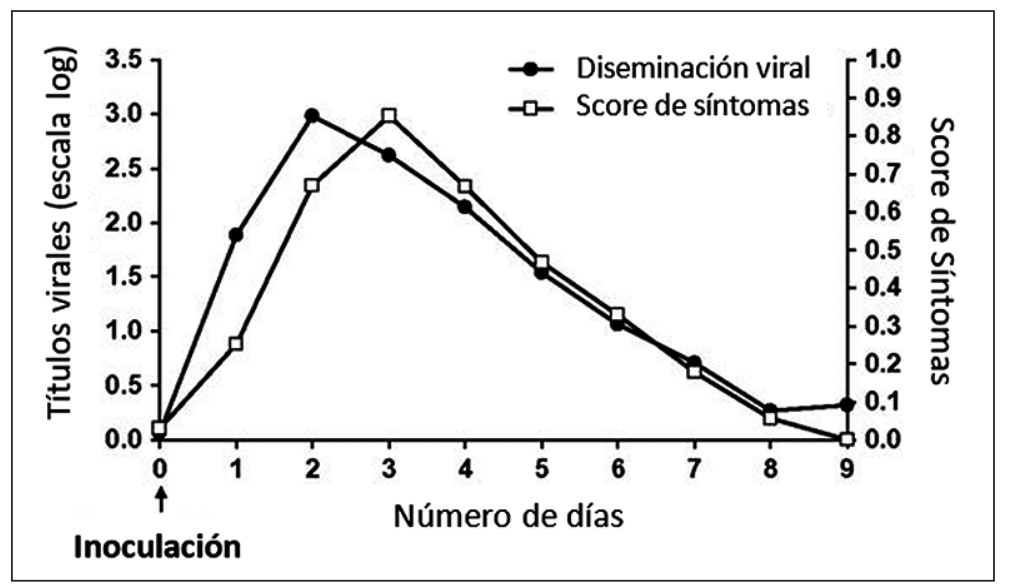

Figura 2. Resumen de curvas de la eliminación del virus y puntajes de síntomas totales en la infección experimental por el virus de la influenza (Adaptado de ${ }^{7}$ ).

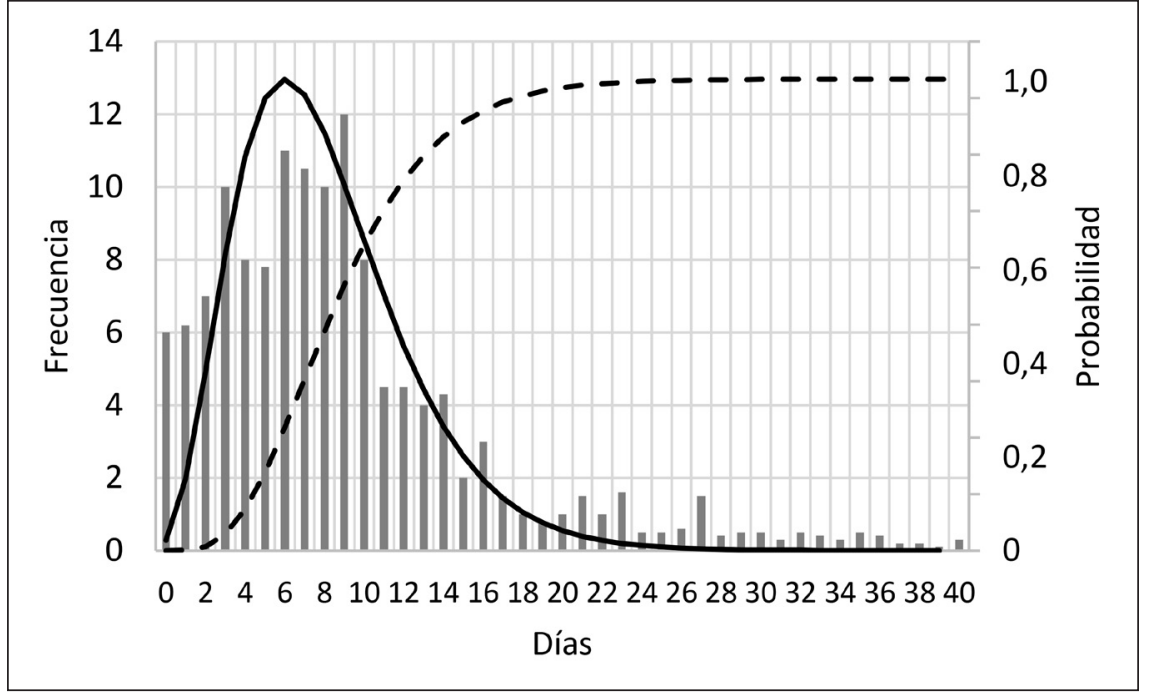

Figura 3. Tiempos observados entre el inicio de la enfermedad asociada al virus Ébola en un paciente de caso índice y el inicio de la enfermedad en la persona infectada (histogramas en gris) y la función de densidad de probabilidad de mejor ajuste (gama) (curva negra) y distribución acumulada (negra punteada) para el período mostrado (Adaptado de ${ }^{8}$ ). en la prensa, usaban este supuesto ${ }^{12,13}$, pero, la función exponencial realmente no describe un brote epidémico ni una epidemia (no obstante que en un inicio sí es una herramienta descriptiva útil), ya que, por construcción, crece hasta el infinito y, en realidad, las epidemias terminan deteniéndose en algún momento, ya sea porque alcanza a contagiar a toda la población susceptible o porque surge alguna medida terapéutica eficaz que lo logra. Al graficar el comportamiento de las epidemias de influenza de 1918 (Gripe española) o del COVID-19 es claro que la función que las describe no es exponencial, sino otra, emparen- tada matemáticamente, pero algo más compleja (Figuras 4 y 5 ).

En realidad, la función que describe el comportamiento de los casos acumulados en las epidemias tiene un componente exponencial que explica las aceleraciones iniciales en la aparición de casos (exponencial con exponente positivo), pero, tiempo después, la función realiza una "torsión" decreciente, también de comportamiento exponencial, pero con exponente de signo negativo para, más adelante, aplanarse y acercarse asintóticamente al total de contagios.

Esta función, con forma sigmoidea similar a 

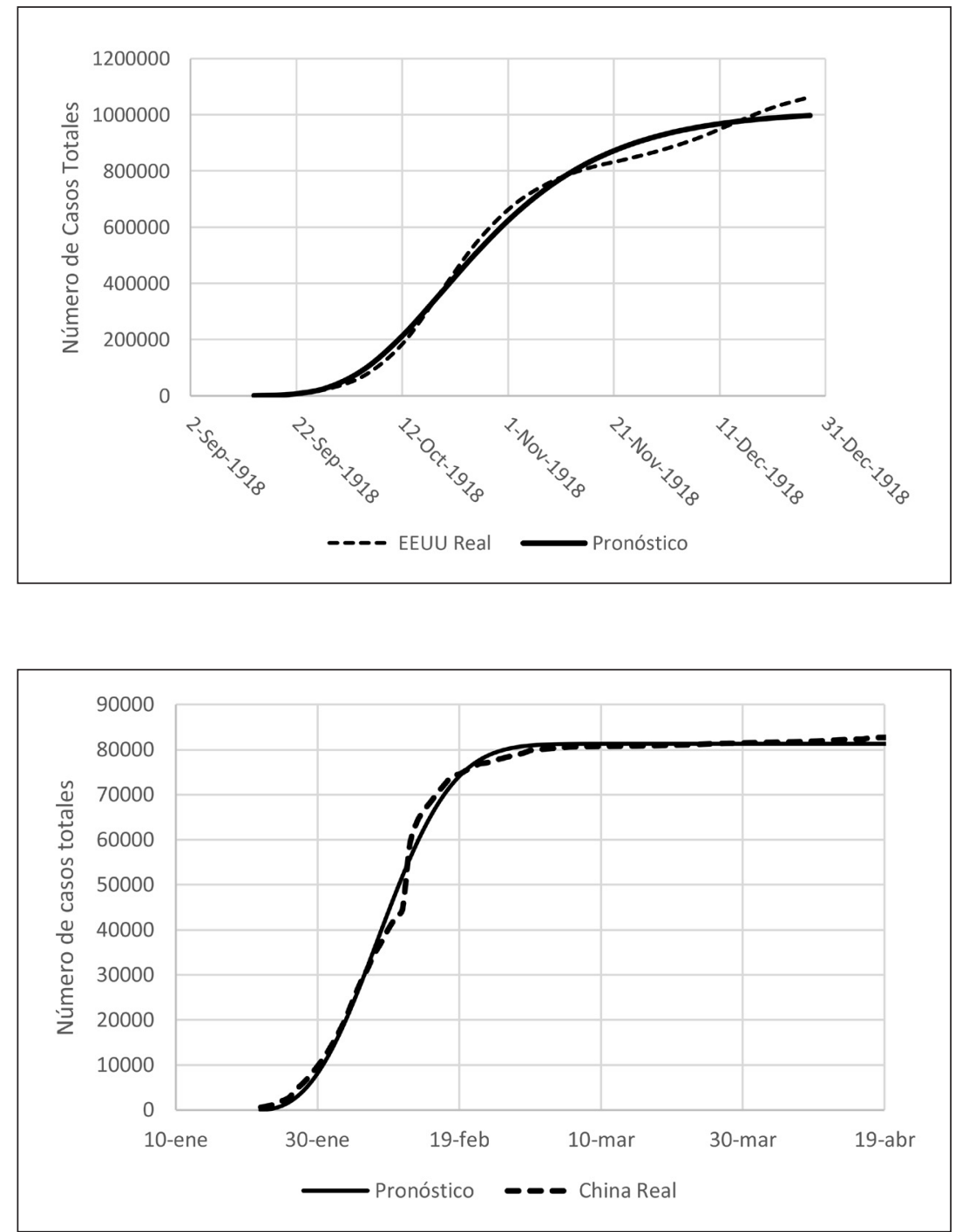

Figura 4. Número de Casos Reales y Pronosticados Totales según función de Gompertz del crecimiento de casos de Influenza en EEUU durante la Pandemia de Gripe Española de 1918. (Adaptado de ${ }^{14}$ ).

Figura 5. Número de Casos Reales y Pronosticados Totales según función de Gompertz del crecimiento de casos de Covid-19 en China durante la Pandemia de 2020. (Adaptado de ${ }^{15}$ ).

la curva de disociación de la hemoglobina puede modelarse con una curva logística o una de Gompertz ("caso particular" de la primera). Ambas suelen utilizárselas para describir el crecimiento de cultivos de microorganismos o de tumores.

Su formulación matemática (Gompertz) es:

$$
y(t)=A^{\star} \exp \left(-\exp \left(-c^{\star}(t-T i)\right)\right.
$$

Siendo " $y(t)$ " el número de casos al tiempo " $\mathrm{t}$ ", " $\mathrm{A}$ " la asíntota superior o número máximo de casos, "exp" el número de Euler, "c" una constante de crecimiento, "Tì" el tiempo cuando acontece la inflexión de la función o el punto donde la concavidad superior comienza a hacerse concavidad inferior y " $\mathrm{t}$ " la fecha de medición o el " $\mathrm{x}$ " de la función. El parámetro Ti desplaza la curva hacia la derecha sin modificar su forma y los parámetros A y c modifican la forma ${ }^{16}$ (Figura 6).

La Figura 6 muestra una línea punteada ("Tasa de Crecimiento c") que corresponde a la derivada de la función original subyacente. Esta derivada informa la "velocidad" de crecimiento de ella. Este modelo, a diferencia de otros similares, como el logístico, tiene su punto de inflexión (PI) cerca del $37 \%$ de la asíntota superior o parámetro A, mien- 


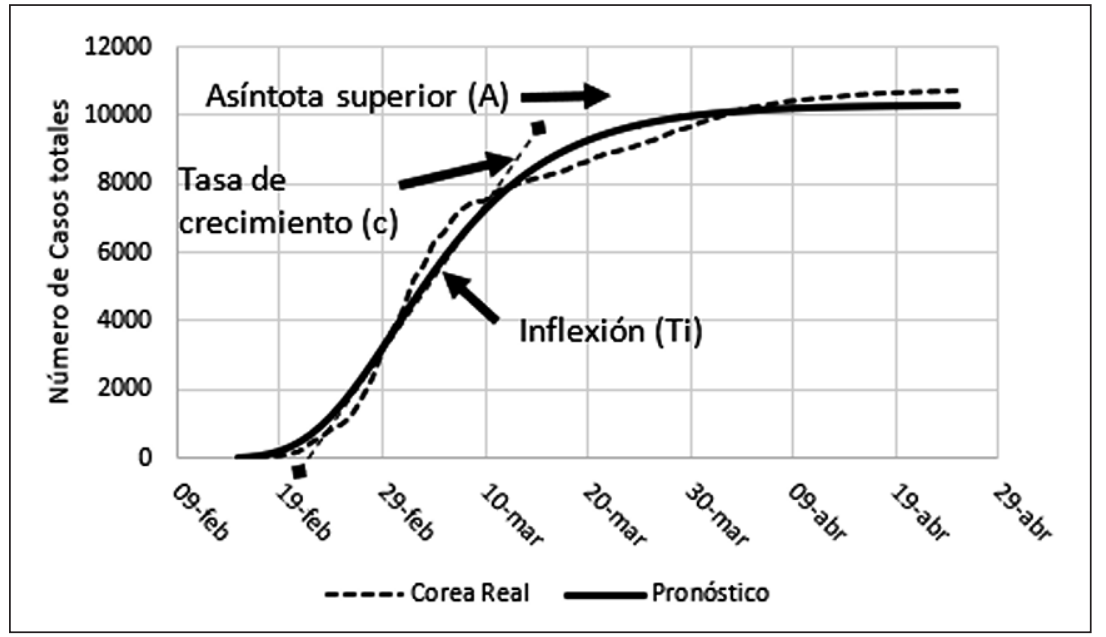

Figura 6. Número de Casos Reales y Pronosticados Totales según función de Gompertz del crecimiento de casos de Covid-19 en Corea del Sur durante la Pandemia de 2020 y Explicación de las componentes de la función de Gompertz (Adaptado de ${ }^{16} \mathrm{y}^{17}$ ). tras que en el logístico se encuentra a mitad de camino entre el mínimo y la asíntota superior $\mathrm{A}^{18}$.

Ahora, ¿de dónde sale R0, entonces? R0 es un "parámetro teórico que proporciona cierta información acerca de la velocidad con la que una enfermedad puede propagarse"19. Puede calculársela de 2 macro formas: con modelos de nivel individual o ILM, y con modelos de nivel poblacional. Los ILM siguen a la persona infectada y su círculo social y requiere de un testeo lo más completo posible para promediar los nuevos casos positivos generados por cada individuo infectado ${ }^{20}$. Los modelos poblacionales, por su parte, usan ecuaciones para modelar la propagación del virus siendo los más famosos: SIR (Susceptible-Infectado-Recuperado) y SEIR (Susceptible-Expuesto-Infectado-Recuperado), usando ecuaciones diferenciales ordinarias (EDOs) para modelar la epidemia. Estos métodos, matemáticamente más o menos complejos, calculan el R0 y otros parámetros útiles, como $\operatorname{Re}(\mathrm{t})$. $\mathrm{R} 0$ es un parámetro estático e independe del tiempo, calculado al inicio del brote, por lo que, si se toman medidas de mitigación de la epidemia, como veremos más adelante, lo correcto será ir calculando el número reproductivo efectivo o Re(t) que sí cambia con el tiempo ${ }^{21}$. La proporción de población susceptible (también variable en el tiempo) al inicio de la epidemia es el parámetro que determina la relación entre R0 $y \operatorname{Re}(\mathrm{t})^{14}$. Ambas metodologías, de aproximación individual y poblacional, no son análogas, porque usan supuestos y técnicas distintas, por lo tanto, sus resultados no son comparables ${ }^{20}$; más aún, es sumamente relevante tener claros los supuestos de cada modelo ya que, de no cumplirse, sesgan sus resultados ${ }^{22}$.

Una forma interesante de estimar R0 es a través del ajuste de un modelo poblacional determinista con la curva de contagios o muertes del brote. La Figura 7 muestra las porciones centrales de las curvas de acumulación de muertes adicionadas al promedio atribuidos a casos de gripe española en varios estados de EEUU ${ }^{14}$. También se muestra las pendientes de las estimaciones lineales de las curvas originales (en escala logarítmica), que dan un promedio de 0,0799 (Rango 0,0568-0,1276) equivalentes (antilogaritmo) a $\mathrm{R}=1,098$ (rango 0,89-1,24).

Esa misma metodología aplicada a los casos de COVID-19 entrega R0 promedio para Nueva Zelanda de 1,83, SurCorea 2,02, China 2,62, Chile 2,62, EEUU 3,47 e Italia 3,60. Más adelante veremos cómo se interpretan estas diferencias de comportamiento matemático del virus en cada país.

Ya sea calculando $\mathrm{R} 0, \operatorname{Re}(\mathrm{t})$ o la derivada de la curva de casos acumulados de infectados, estas "velocidades" irán reflejando período a período cómo se comporta la epidemia y, cuando $\operatorname{Re}(\mathrm{t})$ y/o la velocidad "instantánea" se reduzcan consistentemente, significará que el brote amaina, tal como fue informado para el COVID-19: El R0 "bajó de un 2,6 a un 1,3, lo que explicaría las políticas de volver a los lugares de trabajo y retomar las clases gradualmente"23. 


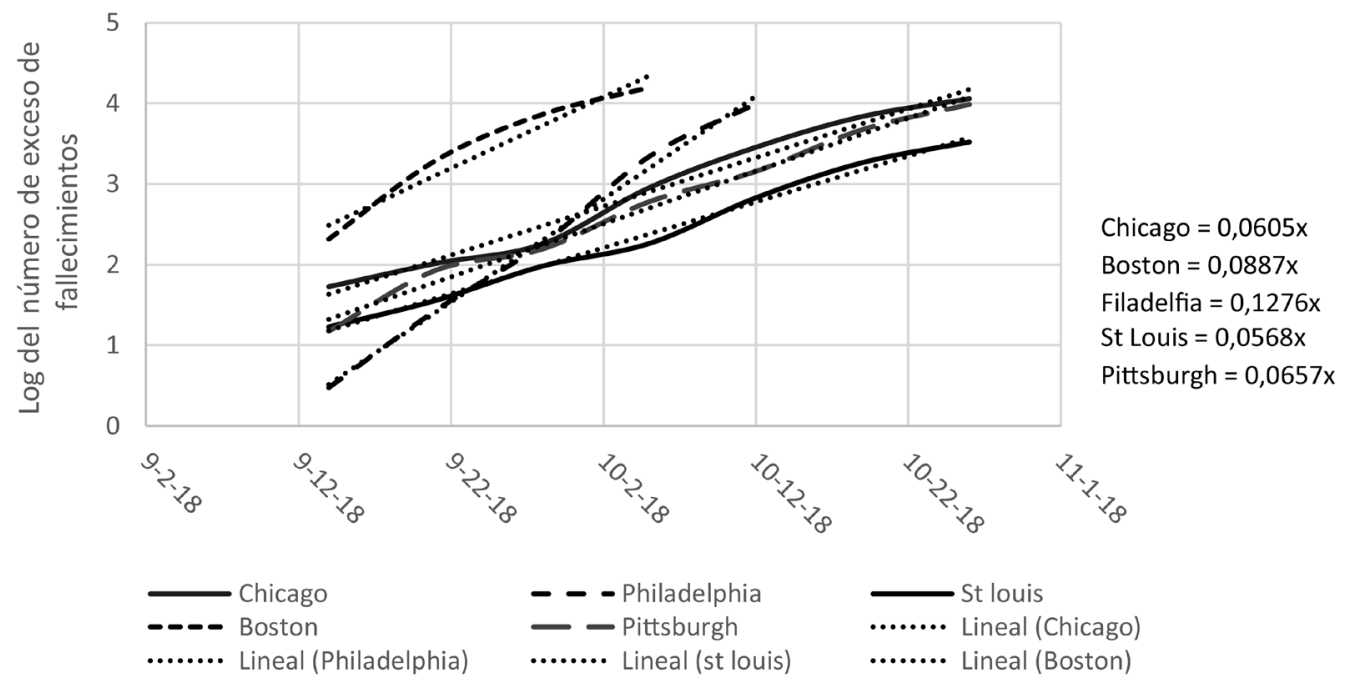

Figura 7. Logaritmo del Número de exceso de muertes y Estimaciones Lineales de la Constante de Crecimiento en varios Estados de EEUU durante la Pandemia de Gripe Española de 1918. (Adaptado de ${ }^{14}$ ).

\section{¿Cuándo se detiene espontáneamente una epidemia?}

Cuando un agente infeccioso nuevo ingresa a una comunidad, infectará progresivamente a todos los susceptibles a una "velocidad" representada por su R0. Sin embargo, en la medida que pasa el tiempo, las personas que se recuperen de ella adquiriendo inmunidad ya no serán capaces de retransmitirla y, por tanto, el $\operatorname{Re}(\mathrm{t})=\mathrm{R} 0$ inicial disminuirá hasta detenerse ${ }^{24}$. Ese umbral de población que "detiene" la infección puede calcularse como 1-1/R0 y origina el concepto de Inmunidad de Rebaño (IR).

La IR representa, entonces, el grado en que la comunidad es susceptible o no a una enfermedad infecciosa como resultado de que miembros de ella hayan adquirido inmunidad, ya sea tras la infección o tras una inmunización profiláctica.

De esta forma, la proporción de la población que habrá contraído la infección cuando ella comience a detenerse, para el sarampión será $92-94 \%$ y para el SARS-CoV-2 60\% $(\mathrm{R} 0=2,5)^{24}$.

Las vacunas confieren inmunidad a quienes las reciben y, con ello, reducen el R0 natural de la infección y el tamaño poblacional que otorga la IR. La Figura 8 ilustra cómo la vacuna de la poliomielitis redujo los casos de infección más allá de lo que se pensaba sólo basado en la eficacia misma de ella ${ }^{25}$.

\section{Estrategias para detener una epidemia}

a. Una epidemia comenzará a descender espontáneamente cuando la IR poblacional alcance su número crítico.

b. Disponer de una vacuna eficaz para alcanzar precozmente la IR. Para que esto sea eficaz, la cobertura de la vacuna debe alcanzar el número de 1-1/R0. Una baja cobertura de los programas de vacunación, por otro lado, puede generar un aumento inadvertido de personas susceptibles dentro la comunidad y serán ellos el posible blanco a sufrir un nuevo brote del agente al haberse reducido el grado de la IR. Ejemplo de esto son los recientes brotes de sarampión en EEUU ${ }^{26}$.

c. No rara vez existe sujetos que desarrollan la enfermedad natural, pero de manera espontáneamente atenuada, aunque pueden, igualmente, contribuir a diseminarla. Si se los identifica y aceptan ser intencionadamente infectados, se podría, teóricamente, aumentar el número de 


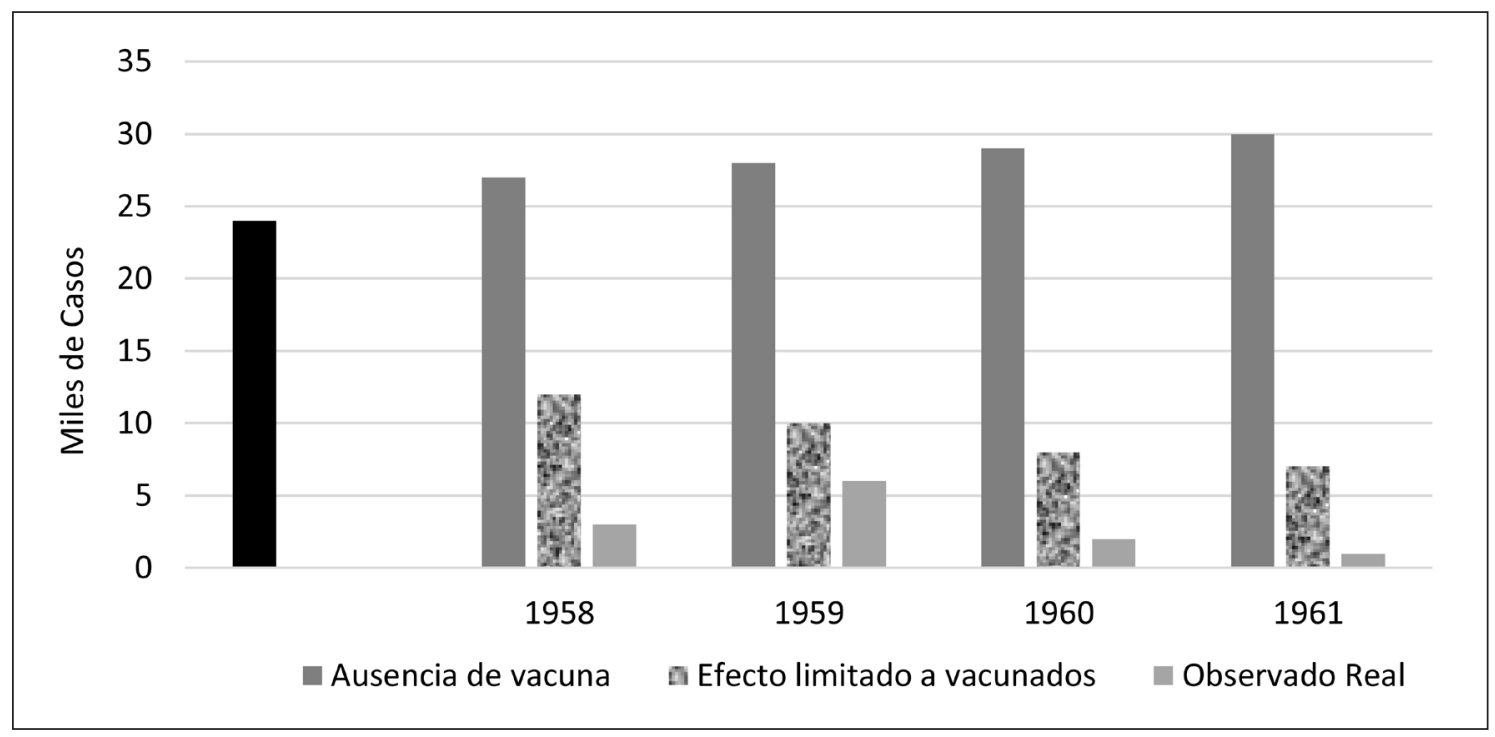

Figura 8. Casos de poliomielitis paralítica esperados en ausencia de vacuna (columnas plomas); con efecto de vacuna limitado a los vacunados (columnas jaspeadas) y casos realmente observados (columnas grises). Promedio de 1951-1954, $1958,1959$. 1960 y 1961 (Adaptado de ${ }^{25}$ ).

personas inmunes y acelerar la llegada a la IR. Si bien esta alternativa puede no ser aceptable, se la empleó previo al registro de la vacuna anti-rubéola. En esa época, se proponía infectar intencionadamente a niñas pequeñas de tal forma que no llegaren a contraer naturalmente la rubéola mientras cursaran un embarazo para, así, evitar la teratogenicidad del virus ${ }^{27}$.

d. Reducir reservorios o interferir con el ciclo de las zoonosis en casos específicos; ejemplos son las infecciones por Arbovirus, como dengue, Zika o chikungunya ${ }^{28}$.

e. Disponer de fármacos que reduzcan la capacidad infectante del virus puede ser considerado como un análogo de la vacuna, ya que reduce la proporción de población susceptible. Ejemplo es dar profilaxis posterior a exposición del $\mathrm{VIH}^{29}$.

f. Usar fármacos que reduzcan el tiempo de contagiosidad y, con ello, el R0 (Figuras 1-3). Ejemplo, oseltamivir e influenza ${ }^{30}$.

g. Enlentecimiento de la vía de contagio que reduce $\operatorname{Re}(\mathrm{t})$ y pendiente media de ascenso del número de casos. Ejemplos:

I. Preservativos para enfermedades de transmisión sexual ${ }^{31}$.

II. Encuestas y testeos de donantes de sangre para evitar infecciones de transmisión sanguínea ${ }^{32}$.
III. Medidas de distanciamiento físico de personas para evitar o, al menos, reducir que secreciones respiratorias de una persona infectada con o sin síntomas contagien a otra susceptible. El resultado esperado es que el número de casos diarios de la enfermedad disminuya en amplitud y se retarde en el tiempo (Figura 9) ${ }^{33}$. Consecuencia de esta estrategia es que se prolongará el lapso de llegada a la IR.

\section{Estrategias de reducción de impacto de la in- fección por COVID-19 en la población}

El R0 promedio para Nueva Zelanda de 1,83, SurCorea 2,02, China 2,62, Chile 2,62, EEUU 3,47 e Italia 3,60 implica que, por cada unidad de tiempo, se diagnosticarán 68, 104, 414, 415, 2941 y 3.999 nuevos contagiados, respectivamente en cada país. Si 4,4\% de los contagiados se hospitaliza y $30 \%$ de éstos necesita cuidados intensivos y ventilación mecánica, puede calcularse la necesidad de disponibilidad de camas críticas y ventiladores mecánicos para evitar que fallezcan enfermos ${ }^{34}$. En Italia, el número de camas críticas diarias que se ocuparían manteniendo el R0 constante $(3,60)$ es $53\left(3.999^{\star} 0,044^{\star} 0,3\right)$, implicando copar 


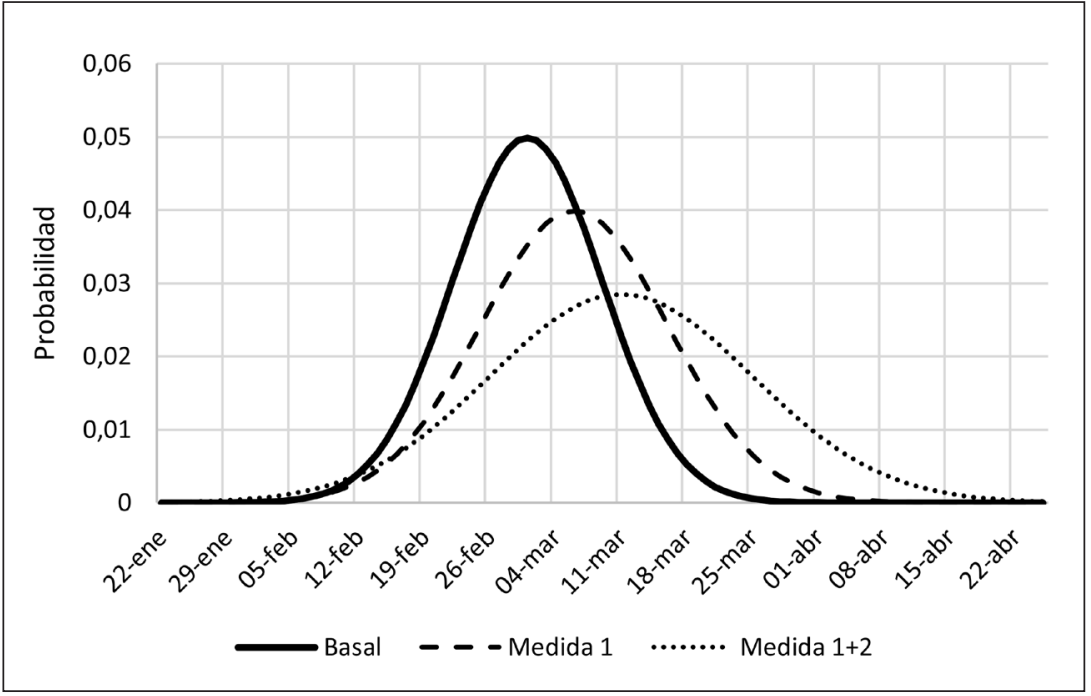

Figura 9. Impacto sobre la densidad de probabilidad de ocurrencia de infección por un agente transmisible al implementar medidas mitigatorias que reduzcan su RO (Adaptado de ${ }^{33}$ ). la disponibilidad total en aproximadamente 100 días (5.324 camas a enero de 2020) ${ }^{35}$, como de hecho aconteción ${ }^{34}$. Por lo tanto, las intervenciones contribuyen a reducir la demanda del recurso terapéutico crítico (ventiladores mecánicos y quienes los manejan), pero, por otro lado, relajar prematuramente las medidas mitigatorias puede generar recrudecimientos en contagios y mortalidad (Figura 10) ${ }^{36}$.

\section{Errores de apreciación}

1. Como bien se ha mostrado, existen muchos factores a considerar a la hora de tomar una medida potencialmente mitigatoria de la expansión de una epidemia. No hacerlo puede llevar a errores y, una medida que aparentemente podría tener sentido, puede terminar demostrando todo lo contrario. En Denver,

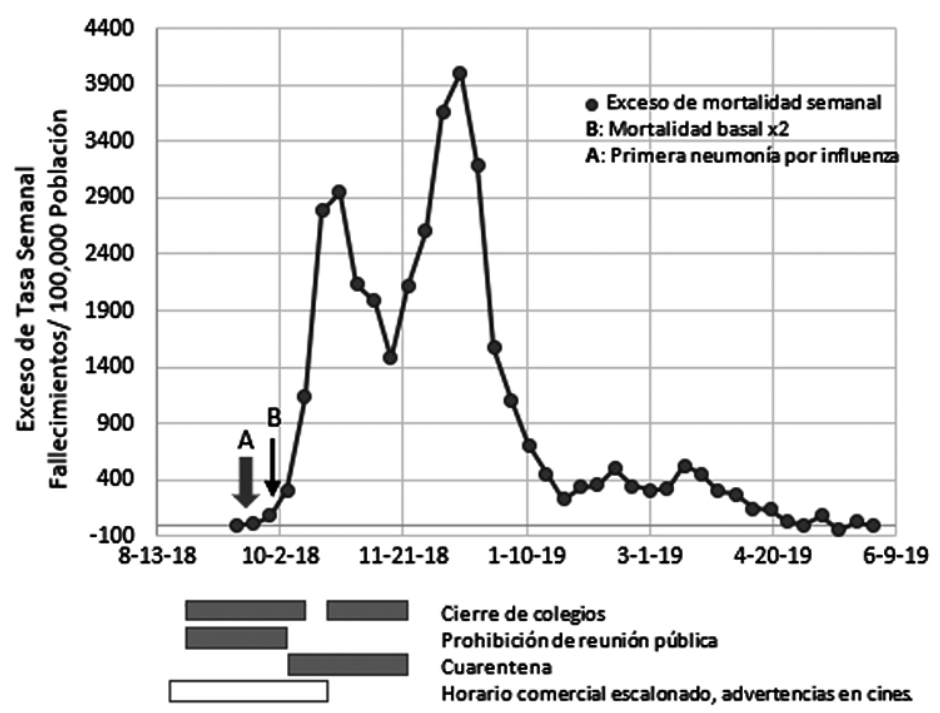

Figura 10. Tasas semanales de exceso de mortalidad desde el 8 de septiembre de 1918 hasta el 31 de mayo de 1919 en Denver, Colorado, durante la pandemia de Gripe Española (Adaptado de ${ }^{37}$ ). 
EEUU, durante la epidemia de gripe española, posiblemente se pensó que "la curva se había aplanado" cuando, probablemente, sólo se encontraban en una sección desacelerada de crecimiento de contagios, y llevó a las autoridades a relajar sus medidas mitigatorias prematuramente, facilitando un segundo brote de influenza (Figura 10) ${ }^{37}$.

2. Basarse sólo en la "inspección visual" de los datos graficados, por ejemplo, mirar la curva logarítmica de contagios acumulados, que busca "normalizar" o "enderezar" la función original, tiende a "adelantar" un aplanamiento de nuevos contagios (Figura 11) ${ }^{38}$. Más aun, sólo emplear transformaciones logarítmicas no es aconsejable debido a que los test estadísticos que se aplican para esta transformación matemática no siempre son válidos para los datos originales $^{39}$.

3. Cuando el $\operatorname{Re}(\mathrm{t})$ o R0 (no todos los medios utilizan bien esta distinción) muestran descensos, significa que se va por el camino del control de la enfermedad. No obstante, considerar cualquier métrica disponible para proponer medidas o incluso juzgar la eficacia de ellas puede ser arriesgado. Como se explicó, debido a que cada forma de calcular $\operatorname{R} 0$ y $\operatorname{Re}(\mathrm{t})$ incorpora muchos y distintos supuestos que deben ser consistentes entre ellos, se hace muy difícil poder compararlos; además, su uso incorrecto produce sesgos ${ }^{22}$.
4. No valerse de las herramientas matemáticas más "simples" disponibles, como la derivada de la función descriptora de contagios acumulados (Figura 7). A diferencia del R0, la derivada no indica a cuántas personas contagia un individuo infectado, pero sí da cuenta de una velocidad relativa de aumento de contagios, por ejemplo, en esa Figura se muestra que la velocidad de crecimiento de contagios en Filadelfia era muy superior a la de Saint Louis, lo cual clarifica las características de la curva y puede ser de gran utilidad para los tomadores de decisión, siendo lo más importante el PI de la curva ( $\mathrm{Ti}$ en Figura 6). Si se conoce la velocidad de la curva y se observa que crece, entonces aún no se llega al PI; mientras que, si se observa que ésta decrece, el PI ya pasó.

5. Más aún, como la curva de Gompertz tiene la particularidad que el PI se alcanza al 37\% de la asíntota superior, si ya se pasó ese punto, para llegar a la IR, o asíntota superior, aún debe pasar casi el doble de tiempo del que transcurrió hasta llegar a ese punto. Del mismo modo, si la función que describe la epidemia es una logística, cuyo PI, tal como se describió más arriba, ocurre al 50\% de su marcha, si se relajan las medidas mitigatorias en el 37\% de la de Gompertz llevará, inevitablemente, a un segundo brote de contagios. Cada medida que se toma altera al menos un parámetro de la

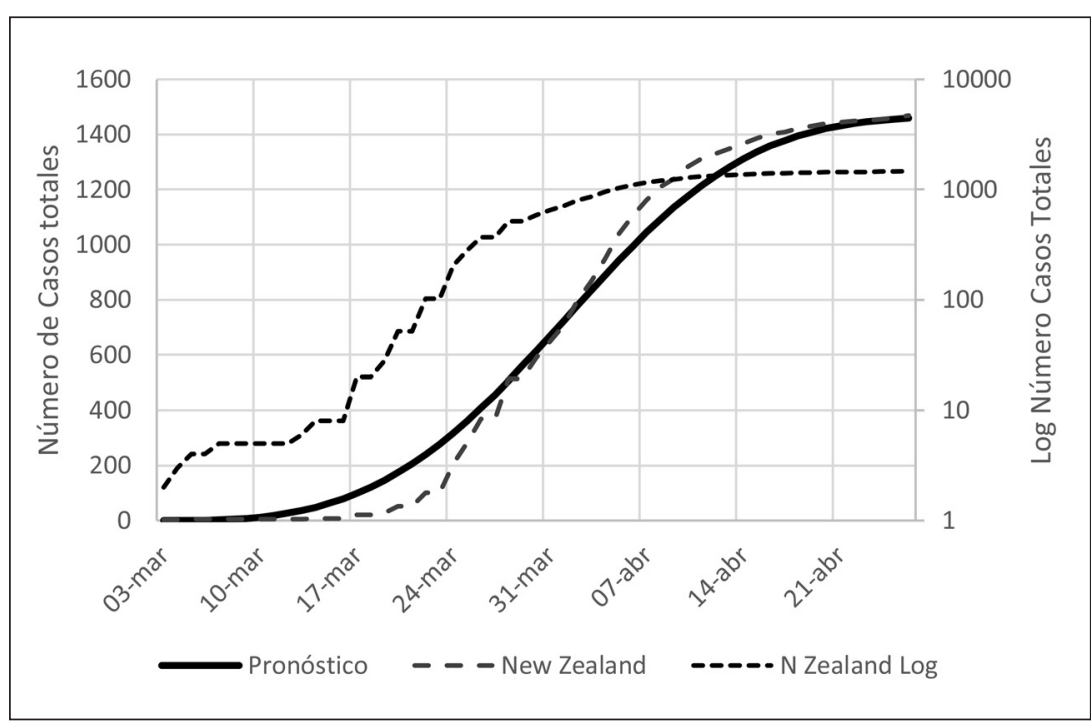

Figura 11. Número de Casos Reales en escala normal y logarítmica y de casos Pronosticados Totales según función de Gompertz del crecimiento de casos de COVID-19 en Nueva Zelanda durante la Pandemia de 2020 y Explicación del engañoso "aplanamiento de la curva" de nuevos contagiados. La línea punteada vertical del 5 de abril muestra cuando el aplanamiento de la curva logarítimica sugiere erradamente que la función original de Gompertz se desacelera (Adaptado de ${ }^{38}$ ). 
ecuación que describe la curva de casos. Por ejemplo, encontrar una vacuna disminuirá el valor de "A", ya que ayuda a llegar a la IR. Medidas de aislamiento social por su parte, podría modificar el valor de "Ti", " $c$ " o " $A$ ".

6. Comparaciones entre las medidas y resultados obtenidos por distintos países frente a un brote epidémico. Al igual que con el $\mathrm{R} 0$, si no se consideran los supuestos del cálculo, las comparaciones pueden ser engañosas. Por ejemplo, los países no comparten densidad ni pirámide poblacional, estructura económica-social, tipo de gobierno, sistema de salud (cobertura y financiamiento), capacidad de atención hospitalaria, cantidad de test realizados, tiempo de respuesta/retraso de resultados, etc. Para hacerlo se necesita un análisis de grupo (cluster analysis) a partir de esas variables ${ }^{40}$.

Enfrentarse a una pandemia es un desafío complejo, que requiere el conocimiento de múltiples factores tanto clínicos, epidemiológicos, como matemáticos, de política pública y económicos. Si éstos no son correctamente aplicados, si no se consideran todos los supuestos ni la fiabilidad de los datos y si se hacen comparaciones sin sustento, se puede cometer errores que podrían costar no sólo algunas, sino muchas vidas humanas. Por esto mismo, los expertos que frecuentemente opinan en medios de comunicación debieren ser cautos en cada una de sus afirmaciones y basar sus opiniones en modelos matemáticos derivados de información empírica y no en sus pareceres y sentires que, muchas veces, pueden ser infundados.

\section{Referencias}

1. WHO | Pneumonia of unknown cause - China [Internet]. WHO. World Health Organization. Disponible en: http://www.who.int/csr/don/05-january-2020-pneumonia-of-unkown-cause-china/en/ [citado el 7 de agosto de 2020].

2. Zhu N, Zhang D, Wang W, Li X, Yang B, Song J, et al. A Novel Coronavirus from Patients with Pneumonia in China, 2019. N Engl J Med 2020; 382 (8): 727-33.

3. Archived: WHO Timeline - COVID-19 [Internet]. Disponible en: https://www.who.int/news-room/detail/2704-2020-who-timeline---covid-19 [citado el 7 de agosto de 2020].
4. Chile Coronavirus: 368,825 Cases and 9,958 Deaths Worldometer [Internet]. Disponible en: https://www. worldometers.info/coronavirus/country/chile/ [citado el 7 de agosto de 2020].

5. Muñoz F. Distribuciones Poisson y Gamma: Una Discreta y Continua Relación. Prospectiva 2014; 12 (1): 99.

6. Paz-Bailey G, Rosenberg ES, Doyle K, Munoz-Jordan J, Santiago GA, Klein L, et al. Persistence of Zika Virus in Body Fluids-Final Report. N Engl J Med 2018; 379 (13): 1234-43.

7. Carrat F, Vergu E, Ferguson NM, Lemaitre M, Cauchemez S, Leach S, et al. Time lines of infection and disease in human influenza: a review of volunteer challenge studies. Am J Epidemiol 2008; 167 (7): 775-85.

8. Ebola Virus Disease in West Africa-The First 9 Months of the Epidemic and Forward Projections. N Engl J Med 2014; 371 (16): 1481-95.

9. Fraser C, Donnelly CA, Cauchemez S, Hanage WP, Van Kerkhove MD, Hollingsworth TD, et al. Pandemic Potential of a Strain of Influenza A (H1N1): Early Findings. Science 2009; 324 (5934): 1557-61.

10. van den Driessche P. Reproduction numbers of infectious disease models. Infect Dis Model 2017; 2 (3): 288-303.

11. Trilla A. Un mundo, una salud: la epidemia por el nuevo coronavirus COVID-19. Med Clin (Barc) 2020; 154 (5): 175-7.

12. Matia Lagos E. En Twitter: «Proyección con los datos actualizados (jueves 19 de marzo de 2020, 11:00 hrs) \#coronavirus \#COVID-19chile \#COVID-19 https://t.co/iCuMv0ut2S»/ Twitter [Internet]. Twitter. Disponible en: https://twitter.com/matialagos/ status/1240641614529339394 [citado el 7 de agosto de 2020].

13. Lillo F. En Twitter: «[4/23] Como podemos ver en el tuit abajo, Chile está recién comenzando. Teníamos 1 infectado el 3 de marzo, y el 10 de marzo ya llegamos a 17. Ese día, utilizando la tasa de crecimiento, proyectamos 30 personas infectadas para el 12 de marzo, y ya confirmaron 33 para hoy. https://t.co/MU9u3tkqbi» / Twitter [Internet]. Twitter. Disponible en: https://twitter.com/ pelaolillo/status/1238116611552395266 [citado el 7 de agosto de 2020].

14. Mills CE, Robins JM, Lipsitch M. Transmissibility of 1918 pandemic influenza. Nature 2004; 432 (7019): 904-6.

15. China Coronavirus: 84,565 Cases and 4,634 Deaths Worldometer [Internet]. Disponible en: https://www. worldometers.info/coronavirus/country/china/ [citado el 7 de agosto de 2020].

16. Tjørve KMC, Tjørve E. The use of Gompertz models in 
growth analyses, and new Gompertz-model approach: An addition to the Unified-Richards family. PLOS ONE 2017; 12 (6): e0178691.

17. South Korea Coronavirus: 14,519 Cases and 303 Deaths - Worldometer [Internet]. Disponible en: https://www. worldometers.info/coronavirus/country/south-korea/ [citado el 7 de agosto de 2020].

18. Winsor CP. The Gompertz Curve as a Growth Curve. Proc Natl Acad Sci 1932; 18 (1): 1-8.

19. Ridenhour B, Kowalik JM, Shay DK. El número reproductivo básico (R0): consideraciones para su aplicación en la salud póblica. Am J Public Health 2018; 108 (Suppl 6): S455-65.

20. Breban R, Vardavas R, Blower S. Theory versus Data: How to Calculate R0? PLOS ONE 2007; 2 (3): e282.

21. Canals LM. Conceptos para una buena toma de decisiones en la pandemia COVID-19 en Chile. Rev Chilena Infectol 2020; 37 (2): 170-2.

22. Obadia T, Haneef R, Boëlle P-Y. The R0 package: a toolbox to estimate reproduction numbers for epidemic outbreaks. BMC Med Inform Decis Mak 2012; 12 (1): 147.

23. Coronavirus: ¿Qué es la tasa de contagio y cómo afecta a la «Nueva normalidad» en Chile? [Internet]. Radio Concierto. 2020. Disponible en: https://www.concierto. cl/2020/04/que-es-la-tasa-de-contagio-y-como-afectaa-la-nueva-normalidad-en-chile/ [citado el 7 de agosto de 2020].

24. Fine PEM, Mulholland K, Scott JA, Edmunds WJ. 77-Community Protection. En: Plotkin SA, Orenstein WA, Offit PA, Edwards KM, editores. Plotkin's Vaccines (Seventh Edition) [Internet]. Elsevier; 2018. p. 15121531.e5. Disponible en: http://www.sciencedirect.com/ science/article/pii/B9780323357616000778 [citado el 7 de agosto de 2020].

25. Stickle G. Observed and Expected Poliomyelitis in the United States, 1958-1961. Am J Public Health Nations Health 1964; 54 (8): 1222-9.

26. Zucker JR, Rosen JB, Iwamoto M, Arciuolo RJ, Langdon-Embry M, Vora NM, et al. Consequences of Undervaccination - Measles Outbreak, New York City, 2018-2019. N Engl J Med 2020; 382 (11): 1009-17.

27. M.D THI. German Measles (1900-1960). Arch Environ Health Int J. 1962; 5 (6): 574-80.

28. Kuno G, Mackenzie JS, Junglen S, Hubálek Z, Plyusnin A, Gubler DJ. Vertebrate Reservoirs of Arboviruses: Myth, Synonym of Amplifier, or Reality? Viruses [Internet] 2017;9(7). Disponible en: https://www.ncbi. nlm.nih.gov/pmc/articles/PMC5537677/ [citado el 7 de agosto de 2020].

29. Drabo EF, Hay JW, Vardavas R, Wagner ZR, Sood N. A
Cost-effectiveness Analysis of Preexposure Prophylaxis for the Prevention of HIV Among Los Angeles County Men Who Have Sex With Men. Clin Infect Dis Off Publ Infect Dis Soc Am 2016; 63 (11): 1495-504.

30. Hayden FG, Atmar RL, Schilling M, Johnson C, Poretz D, Paar D, et al. Use of the Selective Oral Neuraminidase Inhibitor Oseltamivir to Prevent Influenza. N Engl J Med 1999; 341 (18): 1336-43.

31. Steiner MJ, Cates W. Condoms and Sexually-Transmitted Infections. N Engl J Med 2006; 354 (25): 2642-3.

32. Carson JL, Triulzi DJ, Ness PM. Indications for and Adverse Effects of Red-Cell Transfusion. N Engl J Med 2017; 377 (13): 1261-72.

33. Ferguson N, Laydon D, Nedjati Gilani G, Imai N, Ainslie $\mathrm{K}$, Baguelin M, et al. Report 9: Impact of non-pharmaceutical interventions (NPIs) to reduce COVID19 mortality and healthcare demand [Internet]. Imperial College London; 2020. Disponible en: http://spiral.imperial.ac.uk/handle/10044/1/77482 [citado el 7 de agosto de 2020].

34. Redacción. «La sala de emergencias se está derrumbando»: la dramática situación que deben enfrentar los médicos que atienden a pacientes con coronavirus en Italia. BBC News Mundo [Internet]. 19 de marzo de 2020; Disponible en: https://www.bbc.com/mundo/ noticias-51919561 [citado el 7 de agosto de 2020].

35. Sarzanini F. Quanti posti di terapia intensiva ci sono in Italia? (E: bastano?) [Internet]. Corriere della Sera. 2020. Disponible en: https://www.corriere.it/cronache/20_marzo_16/coronavirus-quanti-posti-terapia-intensiva-ci-sono-italia-quanti-ne-arriveranno-0fbafa76-678a-11ea-93a4-da8ab3a8afb1.shtml [citado el 7 de agosto de 2020].

36. Collins SD, Frost WH, Gover M, Sydenstricker E. Mortality from Influenza and Pneumonia in 50 Large Cities of the United States, 1910-1929. Public Health Rep 1896-1970. 1930; 45 (39): 2277-328.

37. Markel H, Lipman HB, Navarro JA, Sloan A, Michalsen JR, Stern AM, et al. Nonpharmaceutical Interventions Implemented by US Cities During the 1918-1919 Influenza Pandemic. JAMA 2007; 298 (6): 644-54.

38. New Zealand Coronavirus: 1,569 Cases and 22 Deaths - Worldometer [Internet]. Disponible en: https://www. worldometers.info/coronavirus/country/new-zealand/ [citado el 7 de agosto de 2020].

39. Feng C, Wang H, Lu N, Chen T, He H, Lu Y, et al. Log-transformation and its implications for data analysis. Shanghai Arch Psychiatry 2014; 26 (2): 105-9.

40. Pacáková V, Papoušková M. Multidimensional Comparisons of Health Systems Functioning in OECD Countries: 7. 